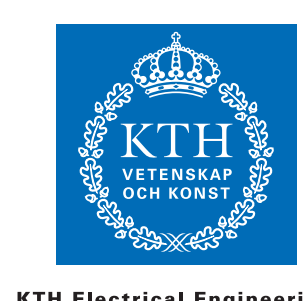

\title{
Optimal Beamforming in MISO Cognitive Channels with Degraded Message Sets
}

C)2012 IEEE. Personal use of this material is permitted. However, permission to reprint/republish this material for advertising or promotional purposes or for creating new collective works for resale or redistribution to servers or lists, or to reuse any copyrighted component of this work in other works must be obtained from the IEEE.

\section{JING LV, RICARDO BLASCO-SERRANO, EDUARD A. JORSWIECK, RAGNAR THOBABEN, AND ADRIAN KLIKS}

Stockholm 2012

Communication Theory Department

School of Electrical Engineering

KTH Royal Institute of Technology 


\title{
Optimal Beamforming in MISO Cognitive Channels with Degraded Message Sets
}

\author{
Jing Lv*, Ricardo Blasco-Serrano ${ }^{\dagger}$, Eduard A. Jorswieck*, Ragnar Thobaben ${ }^{\dagger}$, and Adrian Kliks ${ }^{\ddagger}$ \\ *Communications Theory, Communications Laboratory, Dresden University of Technology, Dresden, Germany \\ E-mail: \{jing.lv, eduard.jorswieck\}@tu-dresden.de \\ ${ }^{\dagger}$ School of Electrical Engineering and ACCESS Linnaeus Centre, KTH Royal Institute of Technology, Stockholm, Sweden \\ E-mail: \{ricardo.blasco, ragnar.thobaben\}@ee.kth.se \\ ${ }^{\ddagger}$ Chair of Wireless Communications, Poznan University of Technology, Poznan, Poland \\ E-mail: akliks@et.put.poznan.pl
}

\begin{abstract}
In this paper we consider the coexistence of a singleinput single-output (SISO) primary link with a multiple-input single-output (MISO) secondary user pair that has non-causal knowledge of the primary message. We study an achievable rate region that exploits this knowledge by combining selfless relaying to maintain the rate supported by the primary link with dirty paper coding to pre-cancel the interference at the secondary receiver. We find the optimal choice of power allocation between these operating modes at the secondary transmitter as well as the optimal beamforming vectors. Moreover, we address the robustness of the solution to uncertainties in the channel knowledge. Finally, we show by numerical evaluation the gains obtained due to the additional knowledge of the primary message.
\end{abstract}

\section{INTRODUCTION}

Consider the coexistence of primary and secondary links in the context of an overlay cognitive radio (CR) system, where the secondary users try to communicate without disturbing the incumbent primary users. In some scenarios it is reasonable to assume that the secondary transmitter has access to the message sent by the primary transmitter in a non-causal fashion. For example, whenever the primary transmitter is much closer to the secondary transmitter than to its intended receiver, the capacity of the channel to the former is much larger than that of the channel to the latter. This allows the secondary transmitter to obtain the message in a fraction of the total transmission time. This channel model is known as the cognitive radio channel or the interference channel (IFC) with degraded message sets [1-5]. The additional knowledge allows for a form of asymmetric cooperation between the two transmitters. For example, one possible strategy is to have the secondary transmitter employ part of its resources to help the communication between the primary users, so that their communication is not disturbed or is even improved (e.g. in terms of rates). The remaining part of the resources are used for private communication to the secondary receiver. Moreover, with accurate channel state information, the secondary transmitter can use its knowledge of the interference experienced by its receiver to pre-cancel it, for example using dirty paper

Part of this work has been performed in the framework of Network of Excellence ACROPOLIS, which is partly funded by the European Union under its FP7 ICT Objective 1.1 - The Network of the Future. coding (DPC) [6]. This strategy was shown to achieve capacity in the weak interference regime in [2] and [3]. In contrast, the capacity of part of the strong interference regime was obtained in [4] using superposition coding and interference decoding. In many scenarios the associated capacity gains over non-cooperative transmission could serve as a motivation for having the primary transmitter share its codebook and message with the secondary transmitter [5].

In a multiuser scenario it is in general desirable that the different users in the system operate efficiently. That is, it should not be possible for individual users to improve their performance (measured, e.g. in terms of rates) unilaterally without degrading that of other users. Such operating points are said to be Pareto optimal. The Pareto boundary for multiple-input single-output (MISO) IFCs was characterized in [7] and [8] for different communication strategies. In general, for underlay CR settings the degradation in terms of quality of service (QoS) to the primary user is a critical aspect. In [9] interference temperature constraints (ITC) [10] were used to measure and limit this effect. In a multipleantenna system, the ITCs are distinguished in soft- and peakpower-shaping constraints [11]. These constraints refer to the maximum average power and average peak power tolerated at the primary receivers, respectively. [12] considers the setting of a single secondary user sharing the same spectral band with multiple primary users. The authors provide optimal transmit beamforming strategies under ITCs for the secondary user. Furthermore, [13] characterizes the Pareto boundary of the MISO IFC through controlling the ITCs at the receivers. Convex optimization techniques for solving CR problems are studied in [14]. The coexistence of a single-input single-output (SISO) primary link and a MISO secondary link is considered in [15], where the achievable secondary rate is optimized under the primary rate constraint, assuming the primary link does not fully load its rate to achieve the Shannon capacity with equality, but there is room that can be exploited by spatial shaping (beamforming) at the secondary transmitter. Moreover, when successive decoding is viable at the secondary receiver, rate splitting at the secondary transmitter increases the achievable secondary rate compared to single-user decoding at the secondary receiver. 


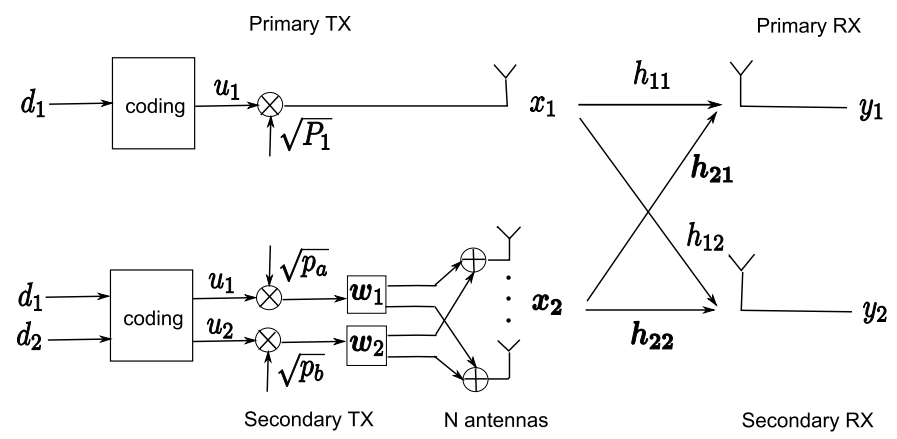

Fig. 1. System model consisting of a SISO primary link and a MISO secondary link.

The contributions of this paper are the following. Firstly, we define an achievable rate region for the MISO cognitive channel based on previous results for the multiple-input multiple-output (MIMO) broadcast channel. Secondly, we find the choice of power allocation and beamforming vectors at the secondary transmitter that maximizes the secondary rate while maintaining a target QoS for the primary users. Then we address the robustness of the beamforming vectors under channel uncertainties. Finally, we evaluate the performance of the proposed scheme numerically, study its behavior for different system parameters, and compare it to an underlay CR system where the secondary transmitter is unaware of the primary message. Our work is related to [16] where the author studied this channel model with a single-stream MIMO secondary user under different CSI assumptions that do not allow for DPC and restricted to zero forcing (ZF) beamforming.

This paper is organized as follows: In Section II we introduce the system model and our problem formulation, alongside with the notation. Section III includes the main contributions of the paper. In Section IV we evaluate numerically the performance of the proposed system for different configurations. Finally, Section V concludes our work.

\section{PRELiminaries}

\section{A. Notations}

Column vectors and matrices are represented in lowercase and uppercase boldface letters, respectively. $|\cdot|$ is the absolute value of a scalar, $\|\cdot\|$ is the Frobenius norm of a vector or matrix, and $(\cdot)^{H}$ stands for Hermitian transpose. $\Pi_{\boldsymbol{X}} \triangleq \boldsymbol{X}\left(\boldsymbol{X}^{H} \boldsymbol{X}\right)^{-1} \boldsymbol{X}^{H}$ denotes the orthogonal projection onto the column space of $\boldsymbol{X}$, and $\Pi_{\boldsymbol{X}}^{\perp} \triangleq \boldsymbol{I}-\Pi_{\boldsymbol{X}}$, where $\boldsymbol{I}$ is the identity matrix, denotes the orthogonal projection onto the orthogonal complement of the column space of $\boldsymbol{X}$. In this paper all rates are expressed in bits.

\section{B. System Model}

The system considered in this paper is depicted in Figure 1 and consists of a SISO primary link and a MISO secondary link with $N$ antennas at the transmitter. The primary transmitter conveys a message $d_{1}$ with rate $R_{1}$ to its intended receiver by encoding it into a codeword $\boldsymbol{x}_{1}$ with an average power constraint $P_{1}$. Similarly, the secondary transmitter wants to convey a message $d_{2}$ with rate $R_{2}$ to its receiver. Since it has non-causal knowledge of the message $d_{1}$, the transmitted codeword $\boldsymbol{X}_{2}$ depends in general on both messages. The secondary transmitter has a total power of $P_{2}$. A fraction $p_{a}$ of it is spent in a selfless manner to help the primary-user pair achieve its rate requirement and the rest of the available power $p_{b}$ (where $p_{a}+p_{b} \leq P_{2}$ ) is used for the transmission of its own message. Furthermore, each of the components has an independent $N$-dimensional beamforming vector $\boldsymbol{w}_{1}$ and $\boldsymbol{w}_{2}$, with $\left\|\boldsymbol{w}_{1}\right\|=\left\|\boldsymbol{w}_{2}\right\|=1$.

The channels are quasi-static block flat fading, and together with the noise components they are modeled as independent and identically distributed complex Gaussian random variables. The channels from the primary transmitter to the primary receiver and the secondary receiver are denoted as $h_{11}$ and $h_{12}$ respectively, with $h_{11} \sim C N(0,1)$ and $h_{12} \sim C N(0, \alpha)$. Similarly, the channels from the secondary transmitter to the primary receiver and the secondary receiver are denoted as $\boldsymbol{h}_{21}$ and $\boldsymbol{h}_{22}$, with $\boldsymbol{h}_{21} \in \mathbb{C}^{N \times 1}$ and $\boldsymbol{h}_{21} \sim$ $C N\left(\mathbf{0}, \beta \boldsymbol{I}_{N}\right), \boldsymbol{h}_{22} \in \mathbb{C}^{N \times 1}$ and $\boldsymbol{h}_{22} \sim C N\left(\mathbf{0}, \boldsymbol{I}_{N}\right)$, where $\mathbf{0}$ and $\boldsymbol{I}_{N}$ denote the all-zero vector and the $N \times N$ identity matrix, respectively. The noise components at the primary and secondary receivers are denoted as $n_{1} \sim C N(0,1)$ and $n_{2} \sim C N(0,1)$, respectively.

\section{Problem Statement}

This work focuses on the interference channel with degraded message sets where a MISO secondary link coexists with a SISO primary link, as described in Section II-B and depicted in Figure 1. First we are interested in defining a set of achievable rates that account explicitly for the dual behavior at the secondary user while maintaining the primary link unchanged.

The resulting rate region allows for a choice of power allocation at the secondary transmitter between the selfish transmission and the cooperation mode, as well as the choice of beamforming vectors. We are interested in finding the parameters that maximize the rate at the secondary transmitter while maintaining a target QoS at the primary receiver measured in terms of achievable rate.

Clearly, utilizing the knowledge of the primary message at the secondary transmitter comes at the price of complexity and overhead. We are interested in the gains obtained by this additional knowledge. Numerical simulations show the impact of the system parameters (number of antennas, signal-to-noise ratio (SNR), and primary link load) on the performance gain.

Finally, the assumption that the channel from the secondary transmitter to the primary receiver is known perfectly is relaxed. This allows us to extend to robust beamforming vectors for spherical uncertainty regions of the aforementioned vector channel. 


\section{MAIN RESUlts}

\section{A. Coding Strategy}

In the overlay $\mathrm{CR}$ context the communication system between the primary user pair is normally given and fixed. Furthermore, we assume that the code used in this link is capacity achieving for a SISO Gaussian channel with a given SNR. At every time instant the message broadcasted by the primary transmitter is

$$
x_{1}=\sqrt{P_{1}} u_{1}\left(d_{1}\right)
$$

where $u_{1}$ is a symbol from the capacity achieving code scaled to have unit average power. Similarly to the work in [2] and [3] (see also [17] and [18]), the secondary user employs superposition coding in combination with DPC [6]. That is, the transmitter employs part of its power to enhance the SNR at the primary receiver. The remaining part of the power is used to convey the message $d_{2}$ to its intended receiver, using DPC to protect it against the interference created due to the transmission of the message intended for the primary receiver. The signal broadcasted by the secondary transmitter at a given time instant is therefore

$$
\boldsymbol{x}_{2}=\sqrt{p_{a}} \boldsymbol{w}_{1} u_{1}\left(d_{1}\right)+\sqrt{p_{b}} \boldsymbol{w}_{2} u_{2}\left(d_{1}, d_{2}\right)
$$

where $u_{2}$ is the corresponding symbol from a capacity achieving DPC scaled to have unit average power.

The above description in terms of $u_{1}$ and $u_{2}$ allows us to write the channel input as

$$
\left[\begin{array}{l}
x_{1} \\
\boldsymbol{x}_{2}
\end{array}\right]=\left[\begin{array}{cc}
\sqrt{P_{1}} & 0 \\
\sqrt{p_{a}} \boldsymbol{w}_{1} & \sqrt{p_{b}} \boldsymbol{w}_{2}
\end{array}\right]\left[\begin{array}{l}
u_{1} \\
u_{2}
\end{array}\right]=\boldsymbol{W} \boldsymbol{u}
$$

where $\boldsymbol{W}$ is a global precoding matrix with size $(N+1) \times 2$. The received signal is

$$
\begin{aligned}
\boldsymbol{y}=\left[\begin{array}{l}
y_{1} \\
y_{2}
\end{array}\right] & =\boldsymbol{H} \boldsymbol{x}+\boldsymbol{n} \\
& =\left[\begin{array}{ll}
h_{11} & \boldsymbol{h}_{21}^{H} \\
h_{12} & \boldsymbol{h}_{22}^{H}
\end{array}\right]\left[\begin{array}{l}
x_{1} \\
\boldsymbol{x}_{2}
\end{array}\right]+\left[\begin{array}{l}
n_{1} \\
n_{2}
\end{array}\right] .
\end{aligned}
$$

This global precoding matrix not only includes the precoding at both transmitters but also reflects the fact that the transmitters are independent and that the primary transmitter has only access to $u_{1}$, while the secondary transmitter has access to both $u_{1}$ and $u_{2}$. The advantage of this seemingly cumbersome notation is that the achievability results from [17] derived for the single-transmitter multiantenna broadcast channel apply directly to our two-transmitter scenario, as long as the precoding matrix is constrained to have the form in (3). Consequently, the following rates are achievable by the primary and secondary users respectively

$$
\begin{aligned}
& R_{1} \leq \log _{2}\left(1+\frac{\left|\sqrt{P_{1}} h_{11}+\sqrt{p_{a}} \boldsymbol{h}_{21}^{H} \boldsymbol{w}_{1}\right|^{2}}{1+p_{b}\left|\boldsymbol{h}_{21}^{H} \boldsymbol{w}_{2}\right|^{2}}\right) \\
& R_{2} \leq \log _{2}\left(1+p_{b}\left|\boldsymbol{h}_{22}^{H} \boldsymbol{w}_{2}\right|^{2}\right)
\end{aligned}
$$

for any choice of precoding vectors $\boldsymbol{w}_{1}, \boldsymbol{w}_{2}$ and powers $P_{1}$, $p_{a}, p_{b}$.

\section{B. Secondary Rate Maximization}

The problem of maximizing the transmission rate $R_{2}$ for the secondary users while satisfying the rate requirement for the primary link $R_{1}$ has the following mathematical formulation:

$$
\begin{aligned}
& \max _{\substack{\boldsymbol{w}_{1}, p_{a} \\
\boldsymbol{w}_{2}, p_{b}}} p_{b}\left|\boldsymbol{h}_{22}^{H} \boldsymbol{w}_{2}\right|^{2} \\
& \text { s.t. } \frac{\left|\sqrt{P_{1}} h_{11}+\sqrt{p_{a}} \boldsymbol{h}_{21}^{H} \boldsymbol{w}_{1}\right|^{2}}{1+p_{b}\left|\boldsymbol{h}_{21}^{H} \boldsymbol{w}_{2}\right|^{2}} \geq 2^{R_{1}}-1 \\
& \qquad\left|\boldsymbol{w}_{1} \|=\right|\left|\boldsymbol{w}_{2}\right| \mid=1 \\
& p_{a}+p_{b} \leq P_{2}, p_{a} \geq 0, p_{b} \geq 0 .
\end{aligned}
$$

Let $h_{11}=\left|h_{11}\right| e^{j \theta}$, we choose

$$
\boldsymbol{w}_{1}=\frac{\boldsymbol{h}_{21}}{\left\|\boldsymbol{h}_{21}\right\|} e^{j \theta}
$$

to maximize left-hand-side of (6b), and have

$$
0 \leq R_{1} \leq \log _{2}\left(1+\left(\sqrt{P_{1}}\left|h_{11}\right|+\sqrt{P_{2}}|| \boldsymbol{h}_{21}||\right)^{2}\right)
$$

where right-hand-side of (8) is achieved by letting $p_{a}=P_{2}$.

From (6b) and (6d) we have $p_{b}=\min \left(P_{2}-p_{a}, \frac{z}{\left|\boldsymbol{h}_{21}^{H} \boldsymbol{w}_{2}\right|^{2}}\right)$ where

$$
z=\frac{\left(\sqrt{P_{1}}\left|h_{11}\right|+\sqrt{p_{a}}|| \boldsymbol{h}_{21}||\right)^{2}}{2^{R_{1}}-1}-1 .
$$

Therefore the formulation in (6) is equivalent to

$$
\begin{aligned}
& \max _{p_{a}} \max _{\boldsymbol{w}_{2}} \min \left(\left(P_{2}-p_{a}\right)\left|\boldsymbol{h}_{22}^{H} \boldsymbol{w}_{2}\right|^{2}, z \frac{\left|\boldsymbol{h}_{22}^{H} \boldsymbol{w}_{2}\right|^{2}}{\left|\boldsymbol{h}_{21}^{H} \boldsymbol{w}_{2}\right|^{2}}\right) \\
& \text { s.t. }\left\|\boldsymbol{w}_{2}\right\|=1,0 \leq p_{a} \leq P_{2} .
\end{aligned}
$$

Similar to the proof of Proposition 1 in [15], given $p_{a}$ the optimal $\boldsymbol{w}_{2}$ can be parametrized as

$$
\boldsymbol{w}_{2}\left(\lambda^{*}\right)=\sqrt{\lambda^{*}} \frac{\Pi_{\boldsymbol{h}_{21}} \boldsymbol{h}_{22}}{\left\|\Pi_{\boldsymbol{h}_{21}} \boldsymbol{h}_{22}\right\|}+\sqrt{1-\lambda^{*}} \frac{\Pi_{\boldsymbol{h}_{21}}^{\perp} \boldsymbol{h}_{22}}{\left\|\Pi_{\boldsymbol{h}_{21}}^{\perp} \boldsymbol{h}_{22}\right\|}
$$

and

$$
p_{b}^{*}=P_{2}-p_{a}
$$

where

$$
\begin{aligned}
& \lambda^{*}= \begin{cases}\lambda_{M R T} & \text { if } \lambda_{M R T} \leq \frac{z}{\left\|\boldsymbol{h}_{21}\right\|^{2}\left(P_{2}-p_{a}\right)} \\
\frac{z}{\left\|\boldsymbol{h}_{21}\right\|^{2}\left(P_{2}-p_{a}\right)} & \text { otherwise }\end{cases} \\
& \lambda_{M R T}=\frac{\left\|\prod_{\boldsymbol{h}_{21}} \boldsymbol{h}_{22}\right\|^{2}}{\left\|\boldsymbol{h}_{22}\right\|^{2}} .
\end{aligned}
$$

(6) can be solved by varying $p_{a}$ from 0 to $P_{2}$ to get the maximum objective value.

Note that the representation in (11) can also be written in terms of a linear combination of maximum ratio transmission (MRT) and ZF as follows [7]

$$
\boldsymbol{w}_{2}\left(\xi^{*}\right)=\frac{\xi^{*} \boldsymbol{w}_{2}^{M R T}+\left(1-\xi^{*}\right) \boldsymbol{w}_{2}^{Z F}}{\left\|\xi^{*} \boldsymbol{w}_{2}^{M R T}+\left(1-\xi^{*}\right) \boldsymbol{w}_{2}^{Z F}\right\|}
$$

with a one-to-one correspondence between $\xi^{*}$ and $\lambda^{*}$, with MRT beamformer $\boldsymbol{w}_{2}^{M R T}=\frac{\boldsymbol{h}_{22}}{\left\|\boldsymbol{h}_{22}\right\|}$ and ZF beamformer $\boldsymbol{w}_{2}^{Z F}=\frac{\Pi_{\boldsymbol{h}_{21}}^{\perp} \boldsymbol{h}_{22}}{\left\|\Pi_{\boldsymbol{h}_{21}}^{\perp} \boldsymbol{h}_{22}\right\|}$. 


\section{Robust Beamforming}

We now assume that the secondary transmitter does not know the channel $\boldsymbol{h}_{21}$ perfectly. In the spherical uncertainty model (a special case of the model in [19]), the channel estimation error is defined as

$$
\boldsymbol{\delta}=\boldsymbol{h}_{21}-\tilde{\boldsymbol{h}}_{21}, \quad \boldsymbol{\delta} \in D(\epsilon),
$$

where $\tilde{\boldsymbol{h}}_{21}$ is the estimate of $\boldsymbol{h}_{21}$ and

$$
D(\epsilon)=\{\boldsymbol{\delta}:\|\boldsymbol{\delta}\| \leq \epsilon\} .
$$

It is most important to satisfy the rate constraint of the primary link even under uncertainty. Therefore the worst-case primary rate can be bounded by finding the strongest possible interference

$$
\max _{\boldsymbol{\delta} \in D(\epsilon)}\left|\left(\tilde{\boldsymbol{h}}_{21}+\boldsymbol{\delta}\right)^{H} \boldsymbol{w}_{2}\right|^{2}=\left(\left|\tilde{\boldsymbol{h}}_{21}^{H} \boldsymbol{w}_{2}\right|+\epsilon\right)^{2}
$$

([20, 21]) and the weakest possible signal

$$
\begin{aligned}
& \min _{\boldsymbol{\delta} \in D(\epsilon)}\left|\sqrt{P_{1}} h_{11}+\sqrt{p_{a}}\left(\tilde{\boldsymbol{h}}_{21}+\boldsymbol{\delta}\right)^{H} \boldsymbol{w}_{1}\right|^{2} \\
& =\left(\left(\left|\sqrt{P_{1}} h_{11}+\sqrt{p_{a}} \tilde{\boldsymbol{h}}_{21}^{H} \boldsymbol{w}_{1}\right|-\sqrt{p_{a}} \epsilon\right)^{+}\right)^{2},
\end{aligned}
$$

where $(x)^{+}=\max \{0, x\}, \forall x \in \mathbb{R}$.

Combining (18) and (19) we obtain the following bound for the quotient in (5a)

$$
\begin{aligned}
& \min _{\boldsymbol{\delta} \in D(\epsilon)} \frac{\left|\sqrt{P_{1}} h_{11}+\sqrt{p_{a}} \boldsymbol{h}_{21}^{H} \boldsymbol{w}_{1}\right|^{2}}{1+p_{b}\left|\boldsymbol{h}_{21}^{H} \boldsymbol{w}_{2}\right|^{2}} \\
& \geq \frac{\left(\left(\left|\sqrt{P_{1}} h_{11}+\sqrt{p_{a}} \tilde{\boldsymbol{h}}_{21}^{H} \boldsymbol{w}_{1}\right|-\sqrt{p_{a}} \epsilon\right)^{+}\right)^{2}}{1+p_{b}\left(\left|\tilde{\boldsymbol{h}}_{21}^{H} \boldsymbol{w}_{2}\right|+\epsilon\right)^{2}} .
\end{aligned}
$$

Please note that the $\mathrm{ZF}$ solution as described in the last subsection, does not work with imperfect channel state information [22]. However, the variation of the power allocation is already included in the optimization algorithm. Hence, the SINR expression from (20) simply replaces the expression in (5a) by

$$
R_{1} \leq \log _{2}\left(1+\frac{\left(\left(\left|\sqrt{P_{1}} h_{11}+\sqrt{p_{a}} \tilde{\boldsymbol{h}}_{21}^{H} \boldsymbol{w}_{1}\right|-\sqrt{p_{a}} \epsilon\right)^{+}\right)^{2}}{1+p_{b}\left(\left|\tilde{\boldsymbol{h}}_{21}^{H} \boldsymbol{w}_{2}\right|+\epsilon\right)^{2}}\right) .
$$

Let $h_{11}=\left|h_{11}\right| e^{j \theta}$, we choose

$$
\boldsymbol{w}_{1}=\frac{\tilde{\boldsymbol{h}}_{21}}{\left\|\tilde{\boldsymbol{h}}_{21}\right\|} e^{j \theta}
$$

to maximize right-hand-side of (21), which becomes

$$
R_{1} \leq \log _{2}\left(1+\frac{\left(\left(\sqrt{P_{1}}\left|h_{11}\right|+\sqrt{p_{a}}\left\|\tilde{\boldsymbol{h}}_{21}\right\|-\sqrt{p_{a}} \epsilon\right)^{+}\right)^{2}}{1+p_{b}\left(\left|\tilde{\boldsymbol{h}}_{21}^{H} \boldsymbol{w}_{2}\right|+\epsilon\right)^{2}}\right)
$$

with

$$
t=\frac{\left(\left(\sqrt{P_{1}}\left|h_{11}\right|+\sqrt{p_{a}}\left\|\tilde{\boldsymbol{h}}_{21}\right\|-\sqrt{p_{a}} \epsilon\right)^{+}\right)^{2}}{2^{R_{1}}-1}-1 \geq 0 .
$$

To maximize $R_{2}$ while satisfying $R_{1}$, similar to the proof of Proposition 1 in [15], given $p_{a}, \boldsymbol{w}_{2}$ can be parametrized as

$$
\boldsymbol{w}_{2}(\lambda)=\sqrt{\lambda} \frac{\Pi_{\tilde{\boldsymbol{h}}_{21}} \boldsymbol{h}_{22}}{\left\|\Pi_{\tilde{\boldsymbol{h}}_{21}} \boldsymbol{h}_{22}\right\|}+\sqrt{1-\lambda} \frac{\Pi_{\tilde{\boldsymbol{h}}_{21}}^{\perp} \boldsymbol{h}_{22}}{\left\|\Pi_{\tilde{\boldsymbol{h}}_{21}}^{\perp} \boldsymbol{h}_{22}\right\|},
$$

where

$$
\lambda^{*}=\tilde{\lambda}_{M R T}=\frac{\left\|\prod_{\tilde{\boldsymbol{h}}_{21}} \boldsymbol{h}_{22}\right\|^{2}}{\left\|\boldsymbol{h}_{22}\right\|^{2}}
$$

and

$$
p_{b}^{*}=P_{2}-p_{a},
$$

if

$$
\tilde{\lambda}_{M R T} \leq \frac{\left(\left(\sqrt{\frac{t}{P_{2}-p_{a}}}-\epsilon\right)^{+}\right)^{2}}{\left\|\tilde{\boldsymbol{h}}_{21}\right\|^{2}},
$$

otherwise, the optimal $\lambda^{*}$ could be found by a line search over $\left[\frac{\left(\left(\sqrt{\frac{t}{P_{2}-p_{a}}}-\epsilon\right)^{+}\right)^{2}}{\left\|\boldsymbol{h}_{21}\right\|^{2}}, \tilde{\lambda}_{M R T}\right]$ to maximize

$$
\frac{\left|\boldsymbol{h}_{22}^{H} \boldsymbol{w}_{2}(\lambda)\right|}{\left|\tilde{\boldsymbol{h}}_{21}^{H} \boldsymbol{w}_{2}(\lambda)\right|+\epsilon}=\frac{\sqrt{\lambda}|| \prod_{\tilde{\boldsymbol{h}}_{21}} \boldsymbol{h}_{22}||+\sqrt{1-\lambda}|| \prod_{\tilde{\boldsymbol{h}}_{21}}^{\perp} \boldsymbol{h}_{22}||}{\sqrt{\lambda}|| \tilde{\boldsymbol{h}}_{21}||+\epsilon}
$$

and

$$
p_{b}^{*}=\frac{t}{\left(\sqrt{\lambda^{*}}|| \tilde{\boldsymbol{h}}_{21} \|+\epsilon\right)^{2}} .
$$

Then $p_{a}$ can be varied from 0 to $P_{2}$ to get the maximum $p_{b}\left|\boldsymbol{h}_{22}^{H} \boldsymbol{w}_{2}(\lambda)\right|^{2}$ while satisfying (24).

\section{NUMERICAL ILLUSTRATIONS}

In the simulation, the achievable secondary rate of the scenario described in Section III-B is shown, together with that of uninformed secondary link without access to the primary message as in [15], with varying SNR and primary link load. For the underlay CR scenario in [15], rate splitting and successive decoding is deployed when it is viable, otherwise singleuser-decoding is used. The load of the primary link is defined as the ratio of primary rate requirement and instantaneously achievable primary rate without secondary transmission. For simulation, we set $P_{1}=10 \mathrm{~dB}$ and $\alpha=\beta=0.5$.

As shown in Figure 2 and 3, for a certain primary link load, secondary antenna configuration, and transmission power, the cooperative transmission strategy derived in this work has a gain over the non-cooperative one in terms of achievable secondary rate. In particular for highly loaded primary links, the gains are tremendous (e.g. for three transmit antennas and 12 dB SNR more than 2 bits per channel use (bpcu), almost a $50 \%$ gain for $100 \%$ load). Interestingly, for three transmit antennas, the achievable rates with informed secondary transmitter (as proposed) with $100 \%$ load are better than with uninformed secondary transmitter with $25 \%$ load. This clearly indicates 


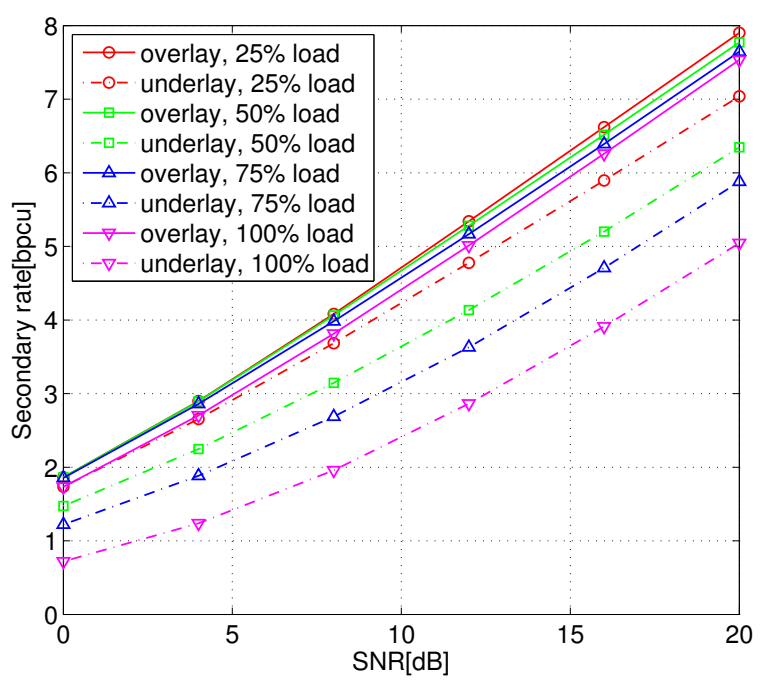

Fig. 2. Achievable secondary rate versus SNR of secondary link: 3 antennas at the secondary transmitter and perfect CSI.

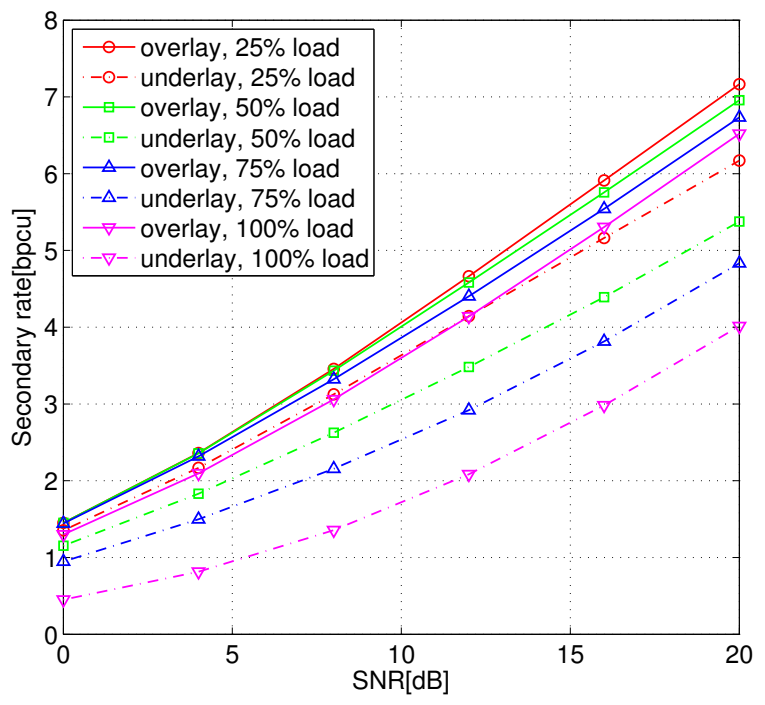

Fig. 3. Achievable secondary rate versus SNR of secondary link: 2 antennas at the secondary transmitter and perfect CSI.

that the knowledge of the primary message at the secondary transmitter helps significantly.

Another observation comparing Figure 2 and 3 is that with sufficient transmit antennas, the performance difference of the informed secondary link with different loads is small (less than $0.5 \mathrm{bpcu})$. Finally, the high SNR slope of the achievable rate of the underlay CR scheme and that of the proposed scheme seem to be different. It would be interesting to study the high SNR behavior of the proposed scheme and compare it with the slope achievable for the completely cooperative scheme, e.g. MIMO with two transmit antennas, which has a slope of

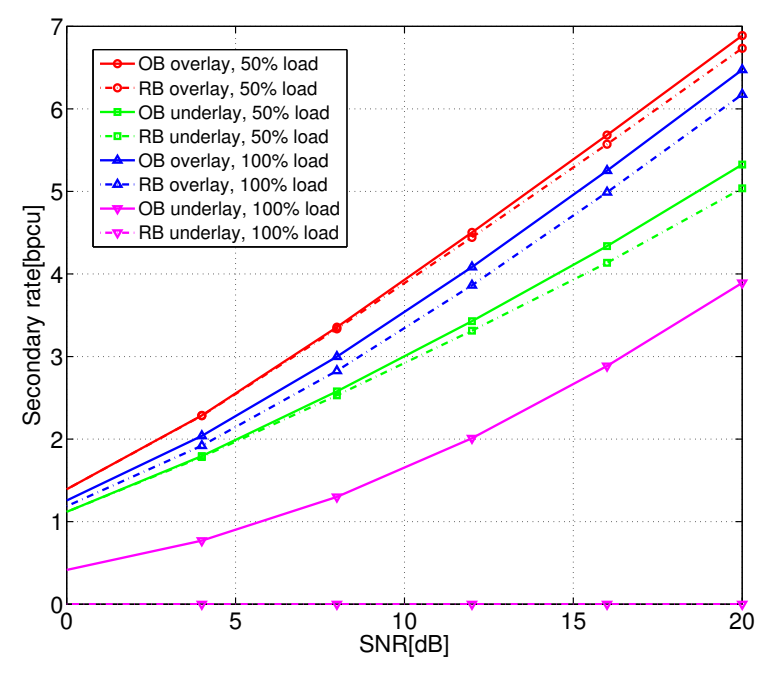

Fig. 4. Achievable secondary rate versus SNR of secondary link: 2 antennas at the secondary transmitter with channel uncertainty.

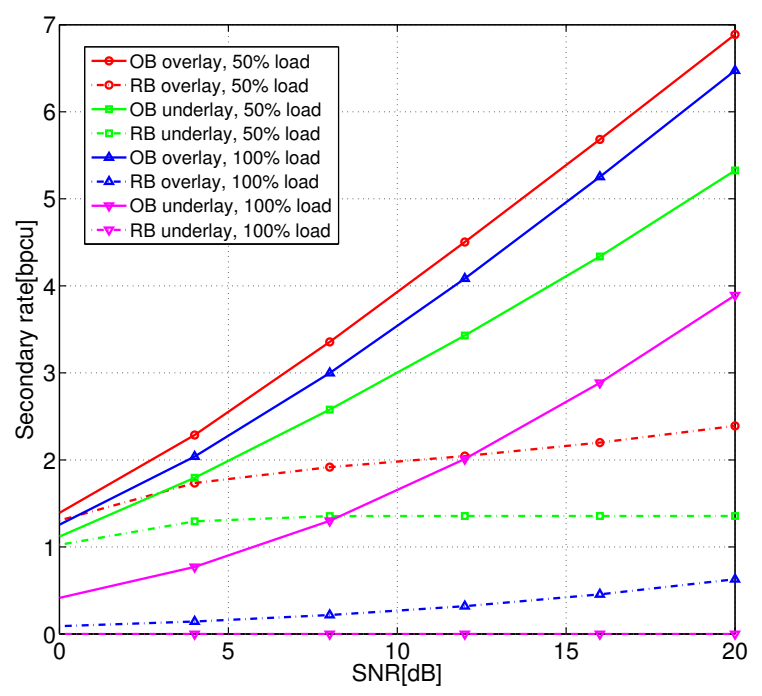

Fig. 5. Achievable secondary rate versus SNR of secondary link: 2 antennas at the secondary transmitter with channel uncertainty.

two.

Finally, the achievable secondary rate of robust beamforming (RB) with channel uncertainty as in Section III-C is compared with that of optimal beamforming $(\mathrm{OB})$ as the proposed scheme in Section III-B. The case of underlay CR scheme in [15] is also included, with the same channel uncertainty model (Similar results can be derived as in Section III-C for both cases of single-user decoding and successive decoding at the secondary receiver.). As seen in Figure 4, when $\epsilon=0.1$, the performance of RB overlay scheme does not change much with respect to OB overlay scheme with $50 \%$ or $100 \%$ load, which nevertheless deteriorates with a greater channel uncer- 
tainty for $\epsilon=1$ as in Figure 5, and deteriorates drastically with $100 \%$ load because the probability is higher that the primary rate requirement is not satisfied. It is similar for the case of $\mathrm{RB} / \mathrm{OB}$ underlay scheme, where $\mathrm{ZF}$ scheme fails with $100 \%$ load because of channel uncertainty.

\section{CONCLUSIONS}

In this paper we have considered the coexistence of a SISO primary link with a secondary MISO user pair that has non-causal knowledge of the message sent by the primary transmitter. Through an appropriate choice of the precoding matrix we have specialized a coding strategy for the MIMO broadcast channel to our scenario. Then we have found the parameters (i.e. power allocation and beamforming vectors) that maximize the rate at the secondary transmitter while ensuring a target QoS for the primary link. In addition, we have analyzed the robustness of our strategy to uncertainties in the knowledge of the channel from the secondary transmitter to the primary receiver. Finally, through numerical analysis we have evaluated the behavior of the system for different configurations (number of antennas, SNR, and primary link load) and we have assessed the gains over underlay CR systems that lack the aforementioned additional knowledge of the primary message.

\section{REFERENCES}

[1] N. Devroye, P. Mitran, and V. Tarokh, "Achievable rates in cognitive radio channels," IEEE Trans. on Information Theory, vol. 52, no. 5, pp. 1813-1827, May 2006.

[2] W. Wu, S. Vishwanath, and A. Arapostathis, "Capacity of a class of cognitive radio channels: Interference channels with degraded message sets," IEEE Trans. on Information Theory, vol. 53, no. 11, pp. 4391 -4399, nov. 2007.

[3] A. Jovicic and P. Viswanath, "Cognitive radio: An information-theoretic perspective," IEEE Trans. on Information Theory, vol. 55, no. 9, pp. 3945 -3958, sept. 2009.

[4] I. Maric, R. Yates, and G. Kramer, "Capacity of interference channels with partial transmitter cooperation," IEEE Trans. on Information Theory, vol. 53, no. 10, pp. 3536 -3548 , oct. 2007.

[5] E. Hossain, L. Le, N. Devroye, and M. Vu, "Cognitive Radio: From Theory to Practical Network Engineering" in New Directions in Wireless Communications Research. Springer, 2009, ch. 10, pp. 251-289.

[6] M. Costa, "Writing on dirty paper (corresp.)," IEEE Trans. on Information Theory, vol. 29, no. 3, pp. 439441, May 1983.

[7] E. A. Jorswieck, E. G. Larsson, and D. Danev, "Complete characterization of the Pareto boundary for the MISO interference channel," IEEE Trans. on Signal Processing, vol. 56, no. 10, pp. 5292-5296, Oct. 2008.

[8] R. Mochaourab and E. Jorswieck, "Optimal beamforming in interference networks with perfect local channel information," IEEE Trans. on Signal Processing, vol. 59, no. 3, pp. 1128-1141, Mar. 2011.

[9] A. Goldsmith, S. Jafar, I. Maric, and S. Srinivasa, "Breaking spectrum gridlock with cognitive radios: An information theoretic perspective," Proc. IEEE, vol. 97, no. 5, pp. 894-914, May 2009.

[10] S. Haykin, "Cognitive radio: brain-empowered wireless communications," IEEE J. Sel. Areas in Commun., vol. 23, no. 2, pp. 201-220, Feb. 2005.

[11] G. Scutari, D. Palomar, J.-S. Pang, and F. Facchinei, "Flexible design of cognitive radio wireless systems," IEEE Signal Process. Mag., vol. 26, no. 5, pp. 107-123, Sep. 2009.

[12] R. Zhang and Y.-C. Liang, "Exploiting multi-antennas for opportunistic spectrum sharing in cognitive radio networks," IEEE J. Sel. Topics in Signal Process., vol. 2, no. 1, pp. 88-102, Feb. 2008.

[13] R. Zhang and S. Cui, "Cooperative interference management with MISO beamforming," IEEE Trans. on Signal Processing, vol. 58, no. 10, pp. 5450-5458, Oct. 2010.

[14] R. Zhang, Y.-C. Liang, and S. Cui, "Dynamic resource allocation in cognitive radio networks," IEEE Signal Process. Mag., vol. 27, no. 3, pp. 102-114, May 2010.

[15] J. Lv and E. A. Jorswieck, "Spatial shaping in cognitive system with coded legacy transmission," in Proc. of International ITG Workshop on Smart Antennas (WSA), Feb. 2011.

[16] U. Salim, "Achievable rate regions for cognitive radio Gaussian fading channels with partial CSIT," in Proc. of International Workshop on Signal Processing Advances in Wireless Communications (SPAWC), June 2011.

[17] G. Caire and S. Shamai (Shitz), "On the achievable throughput of a multiantenna Gaussian broadcast channel," IEEE Trans. on Information Theory, vol. 49, no. 7, pp. 1691-1706, Jul. 2003.

[18] H. Weingarten, Y. Steinberg, and S. Shamai (Shitz), "The capacity region of the Gaussian multiple-input multipleoutput broadcast channel," IEEE Trans. on Information Theory, vol. 52, no. 9, pp. 3936-3964, Sep. 2006.

[19] J. Wang, G. Scutari, and D. P. Palomar, "Robust MIMO cognitive radio via game theory," IEEE Trans. on Signal Processing, vol. 59, no. 3, pp. 1183-1201, Mar. 2011.

[20] S. A. Vorobyov, A. Gershman, and Z.-Q. Luo, "Robust adaptive beamforming using worst-case performance optimization: a solution to the signal mismatch problem," IEEE Trans. on Signal Processing, vol. 51, no. 2, pp. 313-324, 2003.

[21] A. Tajer, N. Prasad, and X. Wang, "Robust linear precoder design for multi-cell downlink transmission," IEEE Trans. Signal Process., vol. 59, no. 1, pp. 235-251, Jan. 2011.

[22] R. Mochaourab and E. A. Jorswieck, "Robust Pareto optimal beamforming in two-user multiple-input singleoutput interference channel," in Proc. of EUSIPCO, 2011. 